\title{
Encoding Reversible Hierarchical Structures with Supramolecular Peptide-DNA Materials
}

\author{
Margaret L. Daly, Yuan Gao, and Ronit Freeman*(-) \\ Department of Applied Physical Sciences, University of North Carolina, Chapel Hill, North Carolina 27599, United States
}

\begin{abstract}
Creating soft materials with the tunable hierarchical structures observed in nature remains an enormous challenge. Synthetic hierarchical systems have been reported, yet strategies to reversibly modulate their structure and function are scarce. We report on the programmable self-assembly of peptide-DNA brush copolymers into supramolecular architectures that can be tuned with changes in temperature, $\mathrm{pH}$, or addition of a soluble trigger. A fiber to bundle transition occurs upon mixing peptides bearing complementary oligonucleotides. These hierarchical structures can be reversed using the programmable nature of

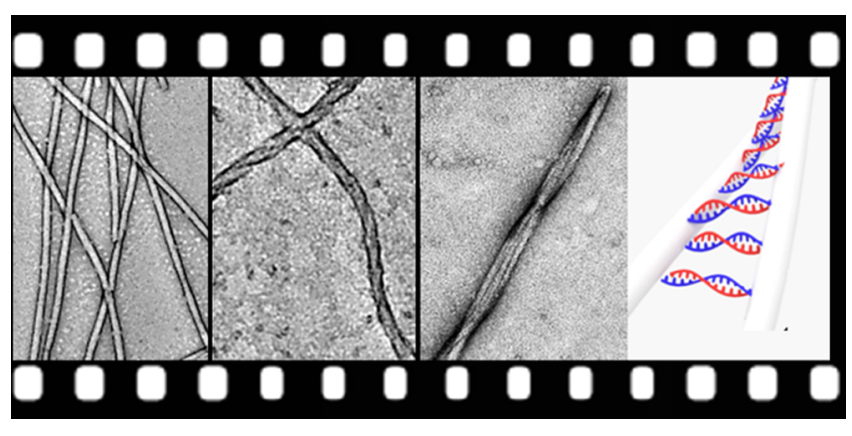
DNA-DNA interactions. The ability to encode the final assemblies in the composition of both amino acid and DNA building blocks provides a strategy for constructing a unique class of reconfigurable supramolecular materials.
\end{abstract}

$\mathrm{H}$ ierarchical supramolecular materials that exhibit order across several length scales are prevalent in nature. ${ }^{1}$ The formation of biological hierarchical structures often involves the organization of soft matter components into nanoscale fibers that then further align, bundle, or wind around each other to form complex high-order architectures such as in bone, ${ }^{2}$ muscle, ${ }^{3}$ and cytoskeleton. ${ }^{4}$ These native fibrous structures can sense and respond dynamically to environmental changes and chemical inputs with a unique capacity for reconfiguration. ${ }^{5}$ Designing molecules that can replicate the multiple levels of structural hierarchy akin to biological systems such as collagen is one of the grand challenges in self-assembly. While approaches to drive the self-assembly of peptides into higher-order structures have been discovered, ${ }^{6-10}$ attaining dynamic and reversible control over hierarchical material formation remains challenging. ${ }^{11}$

DNA-mediated assembly has emerged as a powerful way to program hierarchical architectures but has been primarily confined to inorganic nanoparticles ${ }^{12,13}$ and pure nucleic acid materials. ${ }^{14,15}$ Recently, the first examples of DNA-functionalized supramolecular polymers have been reported, leveraging the structural and functional properties of both synthetic supramolecular polymers and nucleic acids. ${ }^{16-20}$ Yet, the ability to tune and reversibly modulate self-assembled structures, and particularly peptides, using DNA is still in its infancy. ${ }^{11}$ Incorporating dynamic tunability and reversibility to control peptide supramolecular architectures will greatly enhance our ability to design functional soft materials.

We report on grafting DNA strands to short peptides to tune their supramolecular structure and hierarchical organization. The peptide-DNA monomer (pepDNA) consists of a dipeptide sequence covalently linked to single-stranded oligonucleotide segments of various lengths (see the Supporting Information, Scheme S1 and Figures S1-4). For the peptide domain we chose the minimal sequence that is known to self-assemble into high-aspect ratio nanoscale fibers, Fmoc-FF-OH. ${ }^{21,22}$ We hypothesized that a short peptide will be highly dynamic and therefore responsive to DNA-driven manipulations and other environmental triggers with high efficiency. For the DNA segment, we decided to explore oligonucleotides with lengths effective in forming stable duplexes in a cellular or tissue environment $\left(\mathrm{Tm}>37{ }^{\circ} \mathrm{C}\right.$ ) (Table S1). We first synthesized a pepDNA monomer with an oligonucleotide length of 19 bases (pepDNA 19 ) using copperfree click chemistry. ${ }^{23,24}$ Given the large charged domain the DNA building block introduces into the pepDNA monomer, we decided to examine the dependence of structure on the preparative pathway. Freshly prepared aqueous solutions of peptide (P) at $1.87 \mathrm{mM}(0.1 \mathrm{wt} \%)$ contained a polydisperse mixture of fibers and tapes with $20-140 \mathrm{~nm}$ diameter (Figure $1 \mathrm{~A}(\mathrm{i}, \mathrm{iii}))$. The circular dichroism (CD) signal was characteristic of peptides in a $\beta$-sheet secondary structure, with a maximum at $190 \mathrm{~nm}$ and a minimum at $220 \mathrm{~nm}$ (Figure $1 \mathrm{~A}(\mathrm{iv}))$. Interestingly, after annealing the peptide solutions (heating to $95{ }^{\circ} \mathrm{C}$, gently cooling down), transmission electron microscopy (TEM) revealed homogeneous fibers with monodisperse widths of about $30 \mathrm{~nm}$ (Figure 1A(ii,iii)). The

Received: April 13, 2019

Revised: $\quad$ May 21, 2019

Published: June 3, 2019 
A

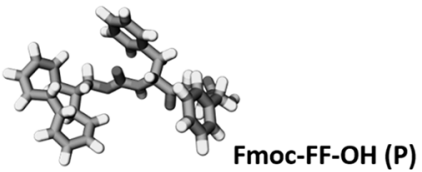

(i)

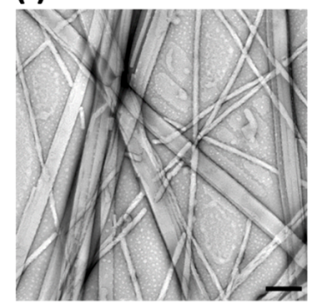

(iii)

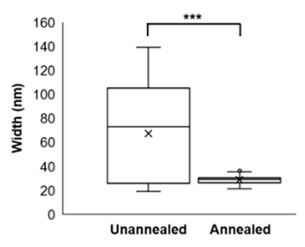

(ii)

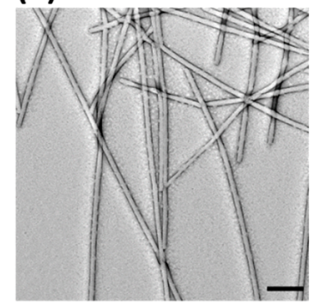

(iv)

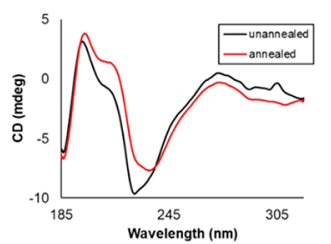

B

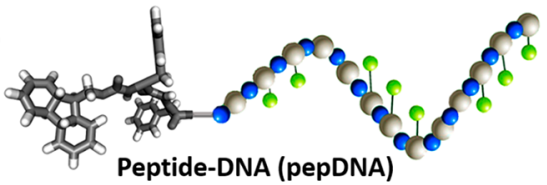

(i)

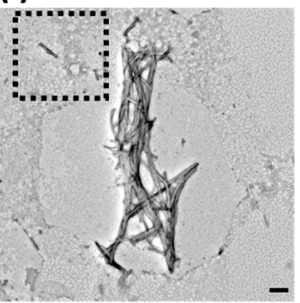

(ii)

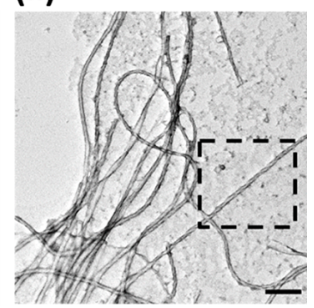

(iv)

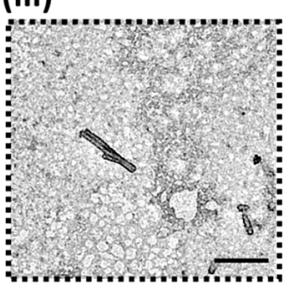

Figure 1. Effect of annealing on $\mathrm{P}$ and pepDNA ${ }_{19}$ morphology. (A) Molecular graphics representation of dipeptide Fmoc-FF-OH (P) and TEM images of the unannealed (i) and annealed (ii) dipeptide assemblies, (iii) Fiber widths as measured by TEM. Box-and-whisker plots show mean and 25 th-75th percentiles from data of $80>\mathrm{Z}$. Statistical analysis was performed using an unpaired two-tailed Student's $t$ test $(* * * p<0.001)$. (iv) CD

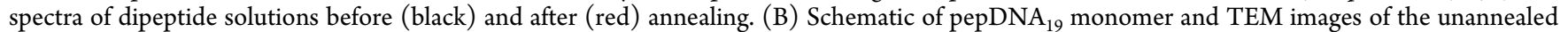
(i) and annealed (ii) pepDNA 19 assemblies. TEM images showing the dominant morphologies in the unannealed (iii) and annealed (iv) pepDNA 19 samples. Scale bars are $200 \mathrm{~nm}$ for all images.

annealed peptides exhibited a CD spectra typical of $\beta$-sheets and signals of $\pi-\pi$ stacking with a red-shifted minimum at 230 $\mathrm{nm}$ and an increased intensity of a maximum at $210 \mathrm{~nm} .^{25}$ The above observations point to the fact that the monodisperse fibers with $\beta$-sheet and $\pi-\pi$ stacking secondary structures are the thermodynamically favored product for P. Next, we studied the effect of the preparative pathway on the self-assembly of pepDNAs. Dissolving pepDNA 19 at $50 \mu \mathrm{M}$ (with $150 \mathrm{mM}$ $\mathrm{NaCl}$ ) resulted in very few supramolecular structures in the form of short filaments $(200 \mathrm{~nm})$ (Figure $1 \mathrm{~B}(\mathrm{i}, \mathrm{iii}))$. However, TEM of the annealed solutions revealed twisted fibers with a $20 \mathrm{~nm}$ diameter and a length much greater than their unannealed counterparts (Figure 1B(ii,iv)). We also found that high ionic conditions are required to screen the negatively charged pepDNAs and allow fiber formation (Figure S5). Taken together, these observations show that the annealing process under high ionic strength conditions enables the rapid growth of long fibers for both the peptide and pepDNA monomers.

We then considered the effect of oligonucleotide length on the supramolecular structure. We synthesized an additional pepDNA monomer with a DNA segment of 46 bases (pepDNA 46 ). Interestingly, varying the length of DNA resulted in fibrous assemblies where the morphology, dimensions, and helicity of the resulting structures were controlled by the length of the DNA domain (Figure 2). Especially surprising is the generation of twisted fibrous structures by annealing solutions of pepDNA ${ }_{46}$, since the fiber-forming peptide motif denotes only $3.5 \%$ of the total molecular weight of the pepDNA monomer. TEM of the annealed peptide and pepDNA assemblies revealed a change in helicity as the number of DNA bases in the monomer increases (Figure 2AC). The pepDNA 19 fibers are an average of $20 \mathrm{~nm}$ wide with a pitch of $147 \mathrm{~nm}$, whereas the pepDNA 46 fibers are an average of $44 \mathrm{~nm}$ wide with a pitch of $632 \mathrm{~nm}$. However, P structures show no twists, suggesting that the DNA block of the pepDNA monomer imparts helicity on the assembled structure and the pitch is dictated by the number of DNA bases in the monomer. Interestingly, the longer DNA domain in pepDNA ${ }_{46}$ promoted a small degree of bundling (Figure 2C), most likely due to short self-complementary domains within the DNA segment. To test this, we monitored the absorbance changes in the DNA spectra in room temperature and upon heating $\left(95^{\circ} \mathrm{C}\right)$ to melt any possible duplex regions. Indeed, we observed a hyperchromic shift indicative of a change from double to single stranded DNA confirmation (Figure S6A), confirming that DNA hybridization drove the formation of pepDNA ${ }_{46}$ bundles. Also, higher salt conditions enabled bundling of pepDNA 46 fibers by further stabilizing interfiber interactions while screening their negative charge, Figure S6B. In addition to tuning the overall morphology, the DNA segment in the pepDNA assemblies is functional and available to bind a complementary strand, as shown in Figure 2F, where the pepDNA $_{19}$ fibers are labeled with dye-modified complementary DNA strands. Since formation of dsDNA introduces additional charges to the already densly charged brush-like supramolecular polymer, we sought to examine what fraction of pepDNA monomers within the fibers can participate in duplex formation under constant salt conditions (150 mM NaCl). By monitoring pepDNA ${ }_{19}$ absorbance upon gradual addition of a complementary strand ( $1-5 \mathrm{~mol} \%$ of pepDNA concentration), we observed a decrease in overall absorbance indicative of formation of a stable dsDNA, Figure S7A. Subsequent addition of DNA (10-25 mol \%) resulted in an increase of overall absorbance, suggesting that the number of formed DNA duplexes has reached saturation. By introducing 

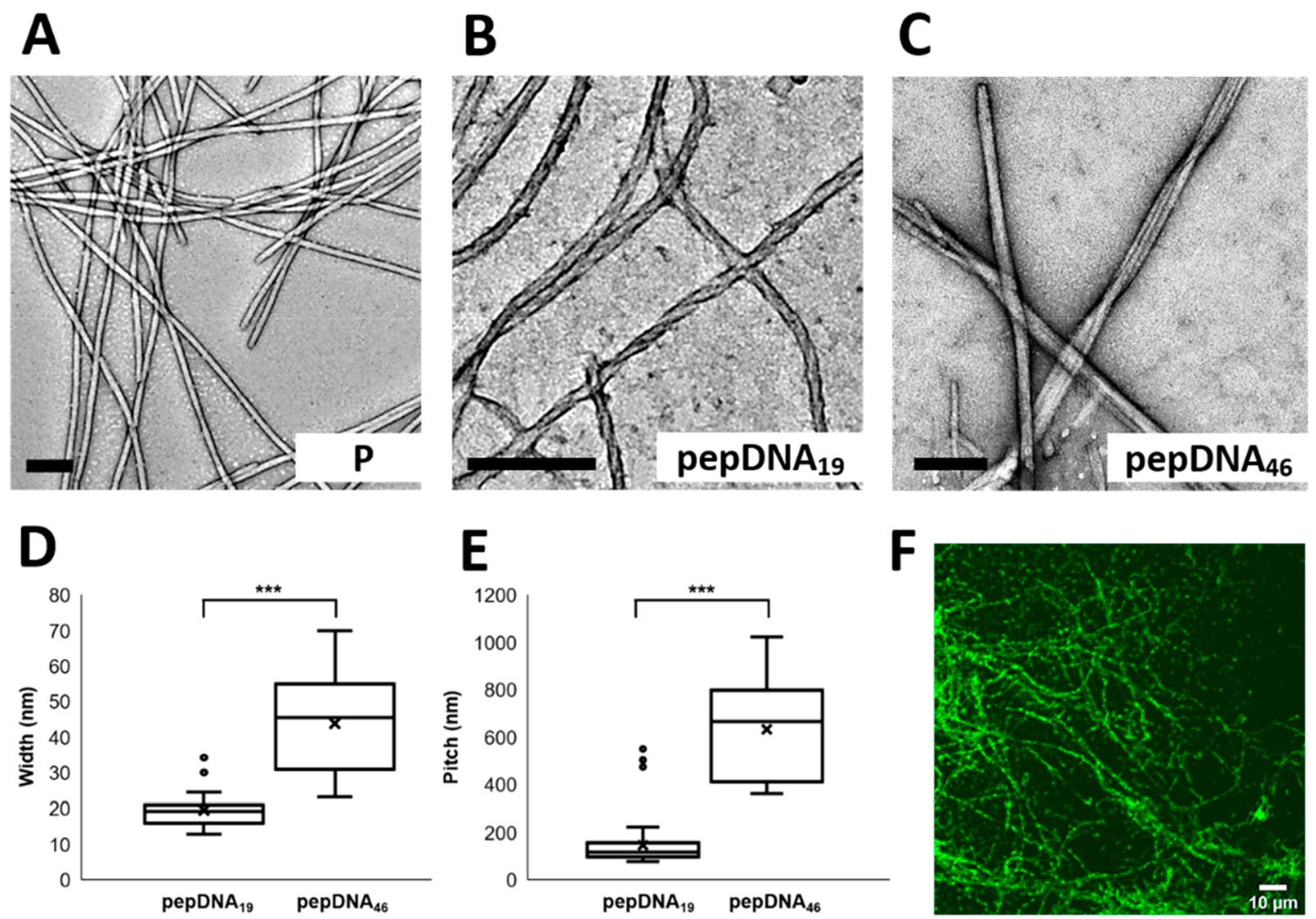

Figure 2. Tuning peptide supramolecular morphology with DNA. (A-C) TEM images of annealed solutions of $\mathrm{P}_{\text {, pepDNA }}$, and pepDNA $\mathrm{p}_{46}$ respectively. Scale bars are $200 \mathrm{~nm}$ for all images. Fiber (D) widths and (E) pitches as measured by TEM. Box-and-whisker plots show mean and 25th-75th percentiles from data of $n>40$. Statistical analysis was performed using an unpaired two-tailed Student's $t$ test $(* * * p<0.001)$. (F) Confocal microscopy image of pepDNA 19 decorated with FAM-modified pepDNA ${ }_{19}$.

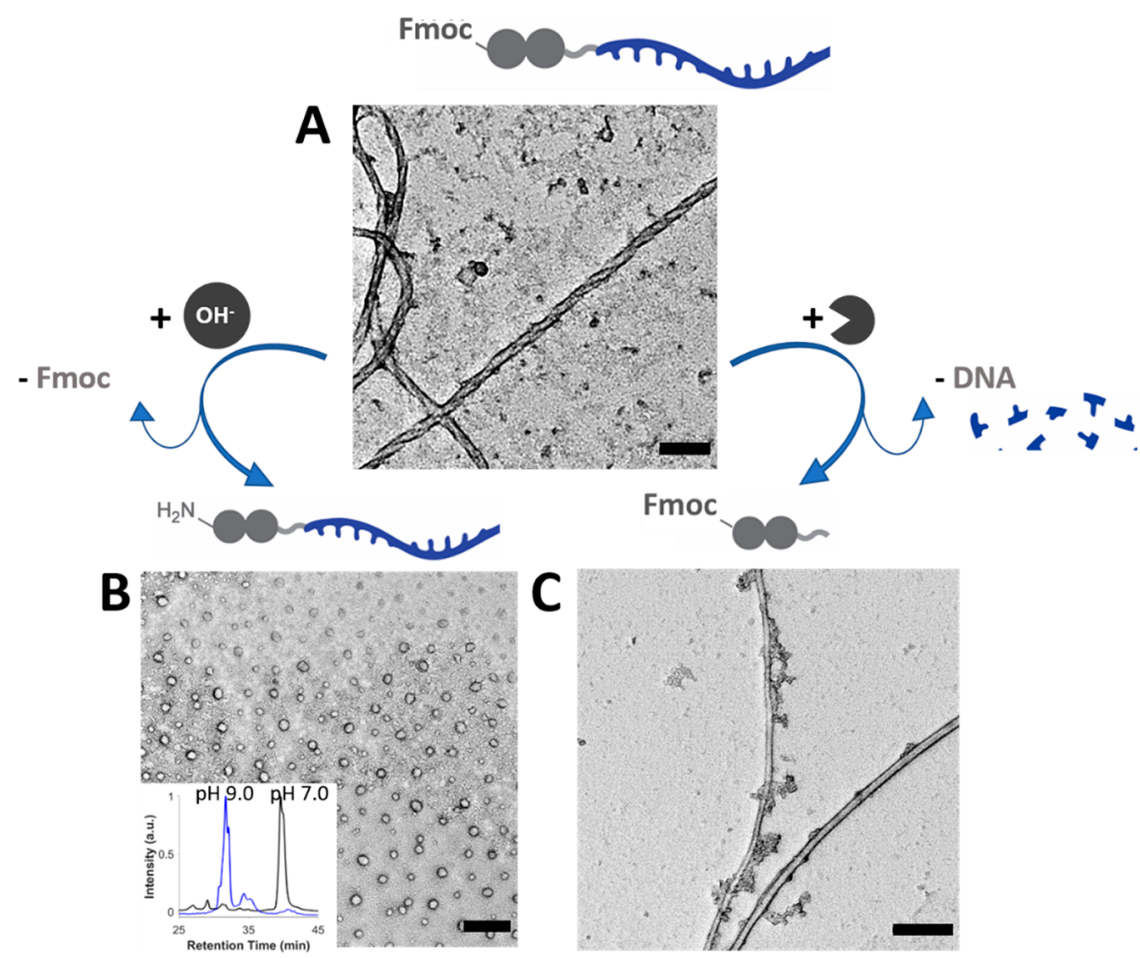

Figure 3. Programming pepDNA morphology with chemical cues. (A) Molecular graphics representation and corresponding morphologies observed by TEM of pepDNA 19 (A) before and (B) after raising the $\mathrm{pH}$ or (C) adding DNase to digest the DNA block. Inset image corresponds to the HPLC chromatograms of pepDNA 19 at $\mathrm{pH} 7.0$ (black) and $\mathrm{pH} 9.0$. (blue). Scale bars are $200 \mathrm{~nm}$ for all images. 


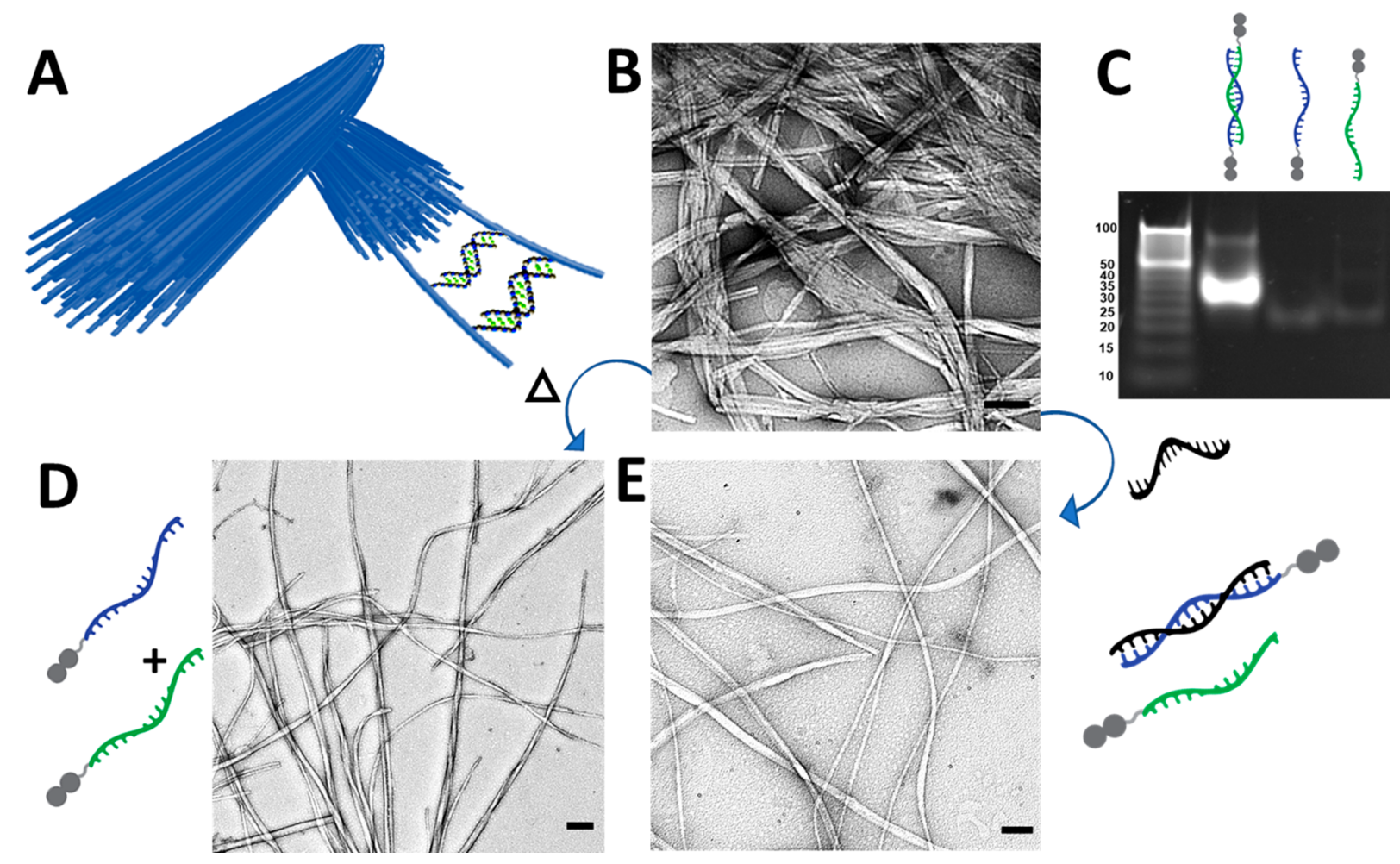

Figure 4. Hierarchical and reversible assembly of pepDNA fibers. (A-C) Hierarchical assembly of pepDNA fibers. (A) Schematic of fiber bundling with DNA handles. (B) TEM image of the annealed complementary assembly. (C) Native polyacrylamide gel of the complementary assembly compared to the individual pepDNA fibers. (D,E) Reversing the hierarchical assembly of pepDNA fibers. (D) Schematic and TEM image of the heated complementary pepDNA assembly. (E) TEM image and schematic of the strand displacement reaction products (E). Scale bars are $200 \mathrm{~nm}$ for all images.

an intercalator dye to a solution containing pepDNA fibers and complementary strands, we were able to determine that approximately $5 \%$ of pepDNA monomers within the fibers existed in duplex form while the remaining unhybridized oligonucleotides persist on the surface of the fibers, Figure S7B,C. The fraction of hybridized DNA segments can be naturally tuned by varying the salt concentration to stabilize additional duplexes.

The ability to tune the supramolecular structure by the length of the DNA segment provides a useful handle to design and control self-assembled architectures. A transition from twisted to linear fibers was observed upon introducing DNase, an enzyme that catalyzes the hydrolytic cleavage of phosphodiester bonds (Figure 3A,C). Cleaving the DNA segment yielded the reorganization of the twisted structure into linear fibers. Another possible handle for supramolecular reconfiguration of the pepDNA is modification of the peptide domain, where the Fmoc group is sensitive to changes in $\mathrm{pH}$. Interestingly, when $\mathrm{pH}$ was raised by adding $\mathrm{NaOH}$, a fiber-tomicelle transition occurred, as revealed by TEM (Figure 3B). MALDI spectra of the solution after $\mathrm{pH}$ was raised indicated the chemical removal of the Fmoc group from the pepDNA monomer (Figure S8). Normalized HPLC chromatograms of the pepDNA solution at $\mathrm{pH} 7.0$ and $\mathrm{pH} 9.0$ (Figure 3B inset) show differences in elution time, in line with a change in the molecular sequence. These results suggest that the Fmoc group was essential to stabilize the pepDNA fiber assembly, and its removal triggered a phase transition into spherical micelles.

Next, we wanted to test if mixing pepDNAs encoded with complementary DNA sequences could direct the assembly of higher-order structures. Ideally, to achieve true biomimetic behavior, such supramolecular architectures will be reversible upon addition of physical or chemical stimuli. The two pepDNA sequences were designed to form a 14-base duplex that is amenable to be reversed upon heating or utilizing DNA strand displacement. ${ }^{26}$ Mixing aqueous solutions of complementary oligonucleotides (pepDNA 19 and pepDNA ${ }_{19}$ ) led to the formation of large twisted bundles of fibers driven by the Watson-Crick base pairing (Figure 4A,B). Native polyacrylamide gel electrophoresis confirmed the formation of larger DNA-directed pepDNA assemblies. (Figure 4C). By monitoring the changes in spectral shape of a DNA intercalator dye added to the pepDNA mixture we found that the resulting bundles were stabilized by about four percent interfiber hybridization between complementary DNA strands, Figure S7C. This suggests that varying the ionic conditions, monomer concentrations or DNA accessibility to increase the number of interfiber interactions, will allow us to systematically tune the dimensions of the resulting fibrous bundles.

We then investigated the reversibility of the hierarchical structure. First, we tested the effect of temperature and found that fibrous bundles could be "melted" at $95{ }^{\circ} \mathrm{C}$. The bundles disappeared, and only individual fibers were visible when the complementary assembly solution was heated and imaged with TEM (Figure 4D). In contrast, when the solution was reannealed, the bundles reformed. The $\mathrm{CD}$ spectra of the complementary assembly at room temperature shows negative peaks at 210 and $240 \mathrm{~nm}$ as well as a broad positive peak at $\sim 280 \mathrm{~nm}$ (Figure S9), indicating the formation of B-form double-stranded DNA. Upon heating, the negative and positive peaks became shallower, suggesting the expected transition to single-stranded DNA. Taken together, these results suggest 
that heating disassembles the interfiber DNA duplexes but upon cooling, the complementary pepDNA monomers rehybridize to recreate the bundles. We also investigated the use of a toehold-mediated strand displacement mechanism to reverse the hierarchical bundled structure. ${ }^{27}$ This was achieved by adding a fully complementary 19-base single-stranded DNA, designed to invade into the shorter interfiber 14-base duplex. As the more thermodynamically stable 19-base duplex forms, the bundles disassemble and the individual fibers reform (Figure 4E). These results demonstrate that the dynamic sequence-specific recognition properties of DNA allow for reversible control of peptide supramolecular structures. This strategy can be extended further by utilizing sequential strand displacement reactions and additional peptide sequences.

Our work shows that combining self-assembled peptides and DNA into the structural design of a material generates various hierarchical supramolecular structures. Interactions between complementary strands on different peptide fibers were used to create higher-order bundled structures. The reversible nature of DNA hybridization and the ability to precisely tune both its affinity and dynamics provides access to modular and uniquely tunable systems that can be applied in tissue engineering, drug delivery, and sensing. At the same time, coupling this capability to the mature field of DNA origami ${ }^{28,29}$ and nanotechnology ${ }^{30}$ will afford additional opportunities to create dynamic hierarchical supramolecular architectures.

\section{ASSOCIATED CONTENT}

\section{S Supporting Information}

The Supporting Information is available free of charge on the ACS Publications website at DOI: 10.1021/acs.bioconjchem.9b00271.

Experimental details and additional figures; synthesis and purification of pepDNAs; TEM of pepDNA assemblies without salt and with high salt content; MALDI spectra of chemical modification to peptide on pepDNA fibers; DNA thermal denaturation in pepDNA fibers; DNA hybridization on pepDNA fibers and in pepDNA bundles; $\mathrm{CD}$ of the complementary pepDNA assembly (PDF)

\section{AUTHOR INFORMATION}

\section{Corresponding Author}

*E-mail: ronifree@email.unc.edu.

\section{ORCID $\odot$}

Ronit Freeman: 0000-0001-5960-6689

\section{Author Contributions}

All authors have given approval to the final version of the manuscript.

\section{Notes}

The authors declare no competing financial interest.

\section{ACKNOWLEDGMENTS}

We thank the Department of Applied Physical Sciences at UNC-Chapel Hill for support of this work. Mass spectrometry was performed at the UNC Mass Spectrometry Core Laboratory at CRITCL (Chemical Research Instrumentation Teaching and Core Laboratories) in the Department of Chemistry. MALDI measurements were done at the UNC Michael Hooker Proteomics Core and CD measurements were taken at the UNC Macromolecular Interactions Facility, where both facilities are of the Lineberger Comprehensive Cancer Center and funded by the National Cancer Institute of the National Institutes of Health (NIH) (P30CA016086). TEM work was performed at the Chapel Hill Analytical and Nanofabrication Laboratory (CHANL), a member of the North Carolina Research Triangle Nanotechnology Network (RTNN) which is funded by the National Science Foundation (NSF) (Grant ECCS-1542015) as part of the National Nanotechnology Coordinated Infrastructure (NNCI). TEM and confocal microscopy were performed at the UNC Hooker Imaging Core (HIC) in the Department of Cell Biology and Physiology. We thank Shimadzu Scientific Instruments and Mrs. Antoinette Swann for the courtesy placement of a full reverse-phase HPLC system to support this research.

\section{REFERENCES}

(1) Fratzl, P., and Weinkamer, R. (2007) Nature's hierarchical materials. Prog. Mater. Sci. 52, 1263-1334.

(2) Weiner, S., and Wagner, H. D. (1998) THE MATERIAL BONE: Structure-Mechanical Function Relations. Annu. Rev. Mater. Sci. 28, 271-298.

(3) Frontera, W. R., and Ochala, J. (2015) Skeletal Muscle: A Brief Review of Structure and Function. Calcif. Tissue Int. 96, 183-195.

(4) Fletcher, D. A., and Mullins, R. D. (2010) Cell mechanics and the cytoskeleton. Nature 463, 485-492.

(5) Wu, H., and Fuxreiter, M. (2016) The Structure and Dynamics of Higher-Order Assemblies: Amyloids, Signalosomes, and Granules. Cell 165, 1055-1066.

(6) Wei, G., Su, Z., Reynolds, N. P., Arosio, P., Hamley, I. W., Gazit, E., and Mezzenga, R. (2017) Self-assembling peptide and protein amyloids: From structure to tailored function in nanotechnology. Chem. Soc. Rev. 46, 4661-4708.

(7) Aida, T., Meijer, E. W., and Stupp, S. I. (2012) Functional supramolecular polymers. Science 335, 813-818.

(8) O’Leary, L. E. R., Fallas, J. A., Bakota, E. L., Kang, M. K., and Hartgerink, J. D. (2011) Multi-hierarchical self-assembly of a collagen mimetic peptide from triple helix to nanofibre and hydrogel. Nat. Chem. 3, 821-828.

(9) Lin, Y. A., Ou, Y. C., Cheetham, A. G., and Cui, H. (2013) Supramolecular polymers formed by ABC miktoarm star peptides. ACS Macro Lett. 2, 1088-1094.

(10) Pappas, C. G., Shafi, R., Sasselli, I. R., Siccardi, H., Wang, T., Narang, V., Abzalimov, R., Wijerathne, N., and Ulijn, R. V. (2016) Dynamic peptide libraries for the discovery of supramolecular nanomaterials. Nat. Nanotechnol. 11, 960-967.

(11) Freeman, R., Han, M., Álvarez, Z., Lewis, J. A., Wester, J. R., Stephanopoulos, N., McClendon, M. T., Lynsky, C., Godbe, J. M., Sangii, H., et al. (2018) Reversible self-assembly of superstructured networks. Science 362, 808-813.

(12) Nykypanchuk, D., Maye, M. M., Van Der Lelie, D., and Gang, O. (2008) DNA-guided crystallization of colloidal nanoparticles. Nature 451, 549-552.

(13) Park, S. Y., Lytton-Jean, A. K. R., Lee, B., Weigand, S., Schatz, G. C., and Mirkin, C. A. (2008) DNA-programmable nanoparticle crystallization. Nature 451, 553-556.

(14) Winfree, E., Liu, F., Wenzler, L. A., and Seeman, N. C. (1998) Design and self-assembly of two-dimensional DNA crystals. Nature 394, 539-544.

(15) Zheng, J., Birktoft, J. J., Chen, Y., Wang, T., Sha, R. Constantinou, P. E., Ginell, S. L., Mao, C., and Seeman, N. C. (2009) From molecular to macroscopic via the rational design of a self-assembled 3D DNA crystal. Nature 461, 74-77.

(16) Li, X., Kuang, Y., Shi, J., Gao, Y., Lin, H. C., and Xu, B. (2011) Multifunctional, biocompatible supramolecular hydrogelators consist only of nucleobase, amino acid, and glycoside. J. Am. Chem. Soc. 133, 17513-17518. 
(17) Vyborna, Y., Vybornyi, M., and Häner, R. (2015) From Ribbons to Networks: Hierarchical Organization of DNA-Grafted Supramolecular Polymers. J. Am. Chem. Soc. 137, 14051-14054.

(18) Noteborn, W. E. M., Saez Talens, V., and Kieltyka, R. E. (2017) Reversible Loading of Nanoscale Elements on a Multicomponent Supramolecular Polymer System by Using DNA Strand Displacement. ChemBioChem 18, 1995-1999.

(19) Wijnands, S. P. W., Engelen, W., Lafleur, R. P. M., Meijer, E. W., and Merkx, M. (2018) Controlling protein activity by dynamic recruitment on a supramolecular polymer platform. Nat. Commun. 9, 65.

(20) Bousmail, D., Chidchob, P., and Sleiman, H. F. (2018) Cyanine-Mediated DNA Nanofiber Growth with Controlled Dimensionality. J. Am. Chem. Soc. 140, 9518-9530.

(21) Reches, M., and Gazit, E. (2005) Self-assembly of peptide nanotubes and amyloid-like structures by charged-termini-capped diphenylalanine peptide analogues. Isr. J. Chem. 45, 363-371.

(22) Smith, A. M., Williams, R. J., Tang, C., Coppo, P., Collins, R. F., Turner, M. L., Saiani, A., and Ulijn, R. V. (2008) Fmocdiphenylalanine self assembles to a hydrogel via a novel architecture based on $\pi-\pi$ interlocked $\beta$-sheets. Adv. Mater. 20, 37-41.

(23) Stephanopoulos, N., Freeman, R., North, H. A., Sur, S., Jeong, S. J., Tantakitti, F., Kessler, J. A., and Stupp, S. I. (2015) Bioactive DNA-peptide nanotubes enhance the differentiation of neural stem cells into neurons. Nano Lett. 15, 603-609.

(24) Freeman, R., Stephanopoulos, N., Álvarez, Z., Lewis, J. A., Sur, S., Serrano, C. M., Boekhoven, J., Lee, S. S., and Stupp, S. I. (2017) Instructing cells with programmable peptide DNA hybrids. Nat. Commun. 8, 15982.

(25) Da Silva, E. R., Walter, M. N. M., Reza, M., Castelletto, V., Ruokolainen, J., Connon, C. J., Alves, W. A., and Hamley, I. W. (2015) Self-Assembled Arginine-Capped Peptide Bolaamphiphile Nanosheets for Cell Culture and Controlled Wettability Surfaces. Biomacromolecules 16, 3180-3190.

(26) Zhang, D. Y., and Seelig, G. (2011) Dynamic DNA nanotechnology using strand-displacement reactions. Nat. Chem. 3, $103-113$.

(27) Zhang, D. Y., and Winfree, E. (2009) Control of DNA Strand Displacement Kinetics Using Toehold Exchange. J. Am. Chem. Soc. 131, 17303-17314.

(28) Wang, Z. G., and Ding, B. (2014) Engineering DNA selfassemblies as templates for functional nanostructures. Acc. Chem. Res. $47,1654-1662$.

(29) Saccà, B., and Niemeyer, C. M. (2012) DNA origami: The art of folding DNA. Angew. Chem., Int. Ed. 51, 58-66.

(30) Seeman, N. C., and Sleiman, H. F. (2018) DNA nanotechnology. Nat. Rev. Mater. 3, 17068. 